\title{
Trazodona: farmacologia e interações medicamentosas
}

\author{
Trazodone: pharmacology and drug interactions
}

\author{
Maria da Graça Cantarelli ${ }^{1}$ \\ Marco Antonio Marcolin²
}

\begin{abstract}
Resumo
Contexto: A trazodona, substância com potencial antidepressivo e ansiolítico, continua sendo uma opção terapêutica, inclusive como indutor do sono. Objetivo: Recuperar informações e buscar novos conhecimentos sobre sua farmacocinética e sua farmacodinâmica e, assim, melhor compreender seu perfil de interações medicamentosas, aumentando a segurança em seu uso. Método: Esta revisão foi realizada por meio de busca eletrônica nos bancos de dados Medline e HighWire Press, utilizando como palavras-chave "trazodone”, "adverse drug reaction" e "drug interaction”. Eventualmente, informações complementares foram extraídas de bibliografia pesquisada e de livros-texto de farmacologia. Resultados: Foram encontradas importantes informações sobre os perfis farmacocinético e farmacodinâmico da trazodona, mostrando o interesse mantido de pesquisadores. Conclusões: A trazodona tem se renovado como uma opção não apenas em depressão e insônia, mas também em certas disfunções sexuais, demonstrando um padrão de interações favorável e um perfil de reações adversas que pode ser contornado se bem avaliado e conduzido.
\end{abstract}

Palavras-chave: Trazodona, farmacologia, interação medicamentosa, reação adversa medicamentosa, citocromo P450.

\begin{abstract}
Background: Trazodone is an atypical antidepressant with further therapeutic effects on anxiety and sleep disorders. Objective: To review the pharmacokinetic and pharmacodynamic properties of trazodone in order to better understand the potential of adverse events related to drug interactions. Methods: Electronic search of Medline and High Wire Press databases using the keywords: "trazodone", "adverse drug reaction", and "drug interaction". Additional references were obtained from pharmacology textbooks. Results: A comprehensive data set on the pharmacological profile of trazodone was obtained. Conclusion: Trazodone is being increasedly prescribed no only to depressive and sleep disorders, but also in certain sexual dysfunctions. The risk of adverse events due to drug interactions is acceptable and manageable, rendering trazodone a useful tool in the treatment of psychiatric and related disorders.
\end{abstract}

Key-words: Trazodone, pharmacology, drug interaction, adverse drug reaction, cytochrome P450.

Recebido: 22/06/2006 - Aceito: 31/08/2006

1 Psiquiatra e internista, pesquisadora do Ambulatório de Depressão do HSL da Pontifícia Universidade Católica do Rio Grande do Sul (PUCRS), professora da pós-graduação em psiquiatria do Instituto Abuchaim, Porto Alegre/RS.

2 Médico-assistente do Instituto de Psiquiatria do Hospital das Clínicas da Faculdade de Medicina da Universidade de São Paulo (HC-FMUSP).

Endereço para correspondência: Maria da Graça Cantarelli. Avenida Carlos Gomes, 281/503 - 90480003 - Porto Alegre, RS. E-mail: mariacantarelli@terra.com.br 


\section{Introdução}

A trazodona, um derivado da triazolopiridina, foi sintetizada na Itália em 1966 e ficou disponível nos Estados Unidos apenas no início dos anos 1980 (Escobar et al., 1980). Foi considerada um dos primeiros antidepressivos de segunda geração, algumas vezes também chamada de atípica, possuindo propriedades farmacológicas e bioquímicas muito específicas e não totalmente compreendidas, continuando a estimular novas pesquisas. Desenvolvida para apresentar efeito antidepressivo, surgiu a partir da hipótese de que a depressão seria causada por um desequilíbrio no mecanismo cerebral responsável pela integração emocional de experiências desagradáveis (Brogden et al., 1981; Golden et al., 2004). Portanto, em sua criação, foram utilizados modelos que mediam a resposta a estímulos ou situações nocivas e desprazerosas. Os resultados obtidos em termos de eficácia antidepressiva e efeito ansiolítico lhe garantiram estar entre os antidepressivos mais prescritos tão logo foi aprovada pela Food and Drug Administration (FDA) norte-americana, apresentando-se como uma alternativa para pacientes intolerantes aos efeitos anticolinérgicos dos clássicos antidepressivos tricíclicos (ATCs).

Por sua capacidade de inibição da recaptação de serotonina, muitas vezes é referida como tal na literatura, embora sua efetividade não pareça se dever a este mecanismo por ser considerado pouco potente (Hingtgen et al., 1984; Liebowitz e El-Mallakh, 1989; Marek et al., 1992; Odagaki et al., 2005). Também apresenta efeito antagonista sobre receptores $5 \mathrm{HT}_{2}$, como a nefazodona, provavelmente ligado aos efeitos terapêuticos dessas substâncias. Em estudos animais, a maioria dos antidepressivos se liga de forma similar aos receptores $5 \mathrm{HT}_{2}$, cujo papel exato no efeito antidepressivo dos fármacos continua alvo de pesquisas (Marek et al., 1992). A eficácia terapêutica da trazodona para depressão mostra-se em muitos estudos comparável à de outros antidepressivos (Brogden et al., 1981; Freemantle et al., 2000; Kasper et al., 2005; Weisler et al., 1994).

\section{Indicações}

A indicação clássica da trazodona continua sendo os quadros depressivos, principalmente quando a ansiedade e a insônia estão presentes (Rotzinger et al., 1998; 1999; Zalma et al., 2000). A dose usual diária varia entre $150 \mathrm{e}$ $200 \mathrm{mg}$, com doses extremas de 50 e $600 \mathrm{mg}$ ( Stefanini et al., 1976; Baldessarini, 2006). Em doses mais baixas, entre 25 e $100 \mathrm{mg}$, tem sido prescrita como hipnossedativo para pacientes com insônia, inclusive quando decorrente da ação de outros antidepressivos, como os inibidores de recaptação de serotonina - ISRS (Dugan, 2001; Golden et al., 2004).

MacHale (2002) reforça a indicação da trazodona no tratamento de depressão em paciente idoso e com doença cardiovascular, considerando os baixos efeitos cardíacos e anticolinérgicos dessa medicação. Ressalva o monitoramento para o desenvolvimento de hipotensão arterial e a eventual necessidade de ajuste de dose para evitar quedas e fraturas ósseas, não raras nesta faixa etária.

Tem sido prescrita para pacientes infectados pelo vírus HIV, demonstrando benefícios decorrentes de sua ação ansiolítica e sedativa, muitas vezes tão necessária (Angelino e Treisman, 2001; Greenblatt et al., 2003). Outras indicações incluem dor neuropática, quadros de agitação em pacientes demenciados, ejaculação precoce e distúrbios eréteis masculinos.

\section{Farmacocinética}

É altamente lipofílica e bem absorvida após administração oral, com pico sangüíneo ocorrendo aproximadamente em uma hora quando ingerida com o estômago vazio e em duas horas quando ingerida com alimento, o que parece não alterar sua biodisponibilidade. Apresenta farmacocinética linear e ligação protéica de $89 \%$ a $95 \%$. Sua eliminação é bifásica, sendo que aproximadamente $1 \%$ é eliminado inalterado na urina. A trazodona é extensivamente metabolizada pelo fígado por meio de N-dealquilação, oxidação e hidroxilação, e uma larga variação interindividual no seu metabolismo tem sido observada (Kasper et al., 2005). Pela N-dealquilação, é formado o metabólito ativo mCPP ( $m$-clorofenilpiperazina), que possui meia-vida mais longa que a substância original. Rotzinger et al. pesquisaram in vitro a participação do sistema citocromo P450 (CYP P450) no metabolismo da trazodona, o que resultou na identificação da enzima CYP 3A4 na formação do mCPP (Rotzinger et al., 1999). Esse achado foi reforçado pela redução da formação desse metabólito na presença de cetoconazol, um conhecido inibidor de CYP 3A4 (Rotzinger et al., 1998). Por sua vez, $1 \%$ do mCPP é eliminado inalterado na urina $\mathrm{e}$ o restante sofre metabolismo ligado à enzima CYP 2D6, sendo que metabolizadores pobres apresentam níveis mais elevados de $\mathrm{mCPP}$.

\section{Farmacodinâmica}

A trazodona parece aumentar a concentração de serotonina extracelular por meio da combinação de mecanismos envolvendo transportadores e receptores de serotonina. A capacidade da trazodona de inibir a recaptação de serotonina foi a primeira explicação para sua ação antidepressiva. Estudos posteriores envolvendo roedores e tecido cerebral humano mostraram entre 25 e 330 vezes maior afinidade por receptores $5 \mathrm{HT}_{2}$ do que para inibição da recaptação de serotonina, enquanto ISRS clássicos, como a fluoxetina, mostram em torno de 15 vezes maior afinidade pela inibição da recaptação de serotonina do que para receptor $5 \mathrm{HT}_{2}$ (Hingtgen et al., 1984).

Trazodona é um forte antagonista de receptor $5 \mathrm{HT}_{2 \mathrm{~A}}$, efeito provavelmente envolvido em sua ação 
antidepressiva e ansiolítica (Stefanini et al., 1976). Também é antagonista de auto-receptores pré-sinápticos $5 \mathrm{HT}_{1}$ ((Stefanini et al., 1976). Seu metabólito ativo mCPP é um potente agonista serotoninérgico direto, agindo sobre os receptores $5 \mathrm{HT}_{2 \mathrm{C}}$, podendo colaborar tanto com os efeitos terapêuticos quanto com os colaterais da trazodona (Kasper et al., 2005).

Marcoli et al. (1998) pesquisaram a ação da trazodona sobre os receptores $5 \mathrm{HT}$ em cerebelo de ratos e verificaram seu potencial de inibição do sistema N-metil-D-aspartato - óxido nítrico - GMPc, interpretado como uma ação que mimetiza a serotonina nessa região. Ao utilizarem antagonistas de receptores $5 \mathrm{HT}$, os resultados foram compativeis com uma ação agonista da trazodona, mais especificamente em receptores $5 \mathrm{HT}_{2 \mathrm{C}}$. Baseando-se em experiências de melhora de quadros de ataxia com a utilização de precursores da serotonina, sugeriram que a trazodona possa ser útil a pacientes com ataxia cerebelar.

Outro receptor investigado foi $5 \mathrm{HT}_{2 \mathrm{~B}}$, altamente expresso em válvulas aórtica e mitral e relacionado à mitogênese, desta vez na busca de maior compreensão sobre os mecanismos pelos quais substâncias como a fenfluramina estão ligadas a doença valvular cardíaca e outras, como a trazodona, não. Neste caso, os autores demonstraram que a ação antagonista da trazodona sobre $5 \mathrm{HT}_{2 \mathrm{~B}}$ contrabalança o efeito agonista do $\mathrm{mCPP}$ sobre o mesmo receptor, aliado ainda ao seu baixo nível sangüíneo, cinco vezes menor que a substância de origem. Esse equilíbrio, portanto, explica o não envolvimento da trazodona, até o momento, com esse tipo de alteração cardíaca (Rothman et al., 2000). Aqui, vemos que a interação entre a substância original e o seu metabólito a partir de suas ações sobre os receptores também influenciará nos efeitos finais de um fármaco.

Uma pesquisa realizada utilizando membranas preparadas a partir de células ovarianas de hamsters chineses demonstrou atividade agonista completa da trazodona e atividade agonista parcial de seu principal metabólito, o mCPP, sobre receptores $5 \mathrm{HT}_{1 \mathrm{~A}}$. Considerando a atividade agonista parcial sobre $5 \mathrm{HT}_{1 \mathrm{~A}}$ de outras substâncias com propriedades ansiolíticas, como a buspirona, os autores trabalharam com a idéia de que, ao menos em parte, a atividade ansiolítica da trazodona possa ser decorrente desta ação sobre o $5 \mathrm{HT}_{1 \mathrm{~A}}$ (Odagaki et al., 2005). Esse efeito ansiolítico tem sido estudado em abusadores de substâncias, com bons resultados (Liebowitz e El-Mallakh, 1989).

Em estudos in vivo, a trazodona é desprovida de atividade anticolinérgica e nas pesquisas clínicas mostra uma incidência similar ao placebo (Golden et al., 2004). Assim, a associação com fármacos que possuam propriedades anticolinérgicas torna-se mais segura, não só do ponto de vista cardíaco, mas também da prevenção de delirium no paciente idoso.

A trazodona fracamente antagoniza receptores adrenérgicos pré-sinápticos do tipo alfa $_{2}$ e é um relativamente potente bloqueador de receptor adrenérgico alfa ${ }_{1}$ pós-sináptico. Enquanto a mianserina mostra uma afinidade equivalente por receptor alfa ${ }_{1}$ e alfa ${ }_{2}$ a trazodona demonstra ser cinco vezes mais ativa sobre alfa (Brogden et al., 1981; Golden et al., 2004).

\section{Interações farmacológicas}

O conhecimento da farmacocinética e da farmacodinâmica dos medicamentos antecede o estudo das interações entre estes. Muitas condições alteram esses mecanismos, tais como o número de combinações envolvidas, as características farmacológicas dos medicamentos em uso, as peculiaridades fisiológicas decorrentes da idade e da herança genética do paciente, as doenças associadas etc. Assim, as informações específicas do indivíduo que está sendo atendido são fundamentais, assim como o conhecimento da farmacologia do medicamento que pretendemos prescrever. Pessoas idosas com múltiplas patologias ou medicações são mais suscetíveis a interações farmacológicas.

As informações sobre interações farmacológicas com a trazodona foram pesquisadas por meio do Medline utilizando as seguintes palavras-chave: "trazodone", "drug interaction", "adverse drug reaction" e "cytochrome P450". Foram também consultadas bibliografias selecionadas e os programas interativos Epocrates e mobileMicromedex. Os resultados encontrados mostraram poucos relatos de interações farmacológicas e a presença de estudos recentes sobre as implicações do sistema citocromo P450 no metabolismo da trazodona e as possíveis interações.

O sinergismo secundário às interações farmacodinâmicas diz respeito principalmente à potencialização do efeito hipotensor e sedativo. Desta forma, o uso combinado de trazodona com anti-hipertensivos e substâncias depressoras do sistema nervoso central (SNC) deve ser monitorado; com clonidina, estão descritos redução do efeito anti-hipertensivo e aumento do risco de depressão do SNC (Brogden et al., 1981; Golden et al., 2004). Entre as substâncias que podem potencializar essa depressão do SNC, estão os barbitúricos, os anti-histamínicos, os opióides, o oxibato de sódio (usado em narcolepsia), a dexmedetomidina (agonista alfa-2 adrenérgico com propriedades sedativas e analgésicas), o aripiprazol, os benzodiazepínicos, os antipsicóticos e outros, incluindo o álcool. Atenção deve ser dada a medicamentos que se apresentem como composições de fármacos, como de acetaminofeno ou de ácido acetilsalicílico com opióides e outros (Epocrates). A possibilidade de acentuar a sonolência, a sedação e o prejuízo psicomotor coloca indivíduos idosos sob maior risco, inclusive de queda e fratura óssea com suas implicações (Golden et al., 2004). Sinérgica também é a interação com bupropiona, aparentemente podendo facilitar a eclosão de crises convulsivas (Epocrates).

Outra interação que pode ser considerada é aquela decorrente da associação com fármacos que causem bloqueio alfa-adrenérgico, mecanismo implicado no 
desencadeamento de priapismo pela trazodona e que também tem sido descrito com outras medicações de forma isolada (ver o subtópico "Priaprismo"); há um potencial teórico e não existe probabilidade de risco para a ocorrência desse tipo de interação, assim como não foram localizados relatos envolvendo trazodona com qualquer uma das medicações citadas.

Há poucas descrições de alterações na anticoagulação decorrentes do uso concomitante de varfarina e trazodona. Foi publicado o caso de uma mulher de 40 anos que apresentou redução de 30\% do tempo de protrombina (TP) e da razão normalizada internacional (RNI) após a introdução da trazodona e retorno aos níveis anteriores com a sua retirada (Hardy e Sirois, 1986). Small et al. (2000) buscaram informações sobre pacientes que haviam recebido trazodona e varfarina entre primeiro de junho de 1998 e trinta de junho de 1999, utilizaram um banco de dados a partir de registros de uma clínica de anticoagulação do Central Texas Veterans Healthcare System e somente foram incluídos pacientes que haviam iniciado trazodona naquele período. Dos 75 pacientes localizados, 3 apresentavam provável interação entre essas substâncias, baseada na escala de probabilidade de Naranjo (Small e Giamonna, 2000). Aqueles que tiveram a trazodona iniciada apresentaram redução do TP e da RNI; quando foi retirada, houve aumento destes níveis. Esses autores citam livros de referência em interação farmacológica que classificam a interação entre trazodona e varfarina como de "início demorado, moderadamente severo e clinicamente não significante”. No entanto, os pacientes que fizerem uso concomitante dessas medicações devem ser mais estreitamente monitorados, tanto na introdução quanto na retirada da trazodona. Não foi encontrado na literatura pesquisada o mecanismo subjacente, ao que parece, ainda desconhecido.

Quadro 1. Interações com a trazodona

\begin{tabular}{|c|c|c|}
\hline Fármaco & Efeito & Referência \\
\hline Clonidina & $\begin{array}{c}\downarrow \text { Efeito anti-hipertensivo } \\
\uparrow \text { Risco de depressão do sistema nervoso central }\end{array}$ & Brogden et al., 1981; Golden et al., 2004 \\
\hline $\begin{array}{l}\text { Barbitúricos } \\
\text { Anti-histamínicos } \\
\text { Opióides } \\
\text { Oxibato de sódio } \\
\text { Dexmedetomidina } \\
\text { Aripiprazol } \\
\text { Benzodiazepínicos } \\
\text { Antipsicóticos } \\
\text { Álcool }\end{array}$ & $\uparrow$ Risco de depressão do sistema nervoso central & Epocrates; Golden et al., 2004 \\
\hline Compostos de acetaminofeno & $\uparrow$ Risco de depressão do sistema nervoso central & Epocrates \\
\hline Compostos de ácido acetilsalicílico & $\uparrow$ Risco de depressão do sistema nervoso central & Epocrates \\
\hline Bupropiona & $\uparrow$ Risco de convulsões & Epocrates \\
\hline Bloqueadores alfa-adrenérgicos & $\uparrow$ Risco de priapismo & Epocrates \\
\hline Varfarina & Pode $\downarrow$ TP e RNI & $\begin{array}{l}\text { Hardy e Sirois, 1986; } \\
\text { Small e Giamonna, } 2000\end{array}$ \\
\hline Medicações serotoninérgicas & $\uparrow$ Risco de síndrome serotoninérgica & $\begin{array}{l}\text { Epocrates; Goldberg e Huk, 1992; } \\
\text { George e Godleski, 1996; Kaneda e } \\
\text { Ohmori, 2001; Kasper et al., 2005; Lacy } \\
\text { e Mathis, 2003; Margolese e Chouinard, } \\
\text { 2000; McCue e Joseph, 2001; Nisijima } \\
\text { et al., 1996; Reeves e Bullen, 1995; } \\
\text { Sternbach, } 1991\end{array}$ \\
\hline Ritonavir & 个 Sedação e fadiga & Greenblatt et al., 2003 \\
\hline Fenitoína & Possível toxicidade & Dorn, 1986 \\
\hline Digoxina & Possível toxicidade & Rauch e Jenike, 1984 \\
\hline Gingko biloba & Relato de coma & Galuzzi et al., 2000 \\
\hline $\begin{array}{l}\text { Imatinibe } \\
\text { Amiodarona }\end{array}$ & $\begin{array}{c}\uparrow \text { Níveis de trazodona } \\
\uparrow \text { Risco de síncope e hipotensão }\end{array}$ & Epocrates \\
\hline Carbamazepina & $\downarrow$ níveis de trazodona & Epocrates \\
\hline
\end{tabular}


Alguns relatos nos mostram que a síndrome serotoninérgica (SS) é uma complicação possível quando a trazodona é utilizada concomitantemente a qualquer medicação capaz de aumentar a transmissão serotoninérgica. $\mathrm{O}$ mecanismo da SS é compreendido como sendo decorrente do excesso de serotonina disponível no SNC atuando em $5 \mathrm{HT}_{1 \mathrm{~A}}$ (Sternbach, 1991). Foram encontrados registros desse tipo de interação na associação de trazodona com paroxetina (Reeves e Bullen, 1995), sertralina (Lacy e Mathis, 2003), fluoxetina (George e Godleski, 1996), venlafaxina (McCue e Joseph, 2001), amitriptilina mais lítio (Nisijima et al., 1996) e nefazodona (Margolese e Chouinard, 2000). Com os IMAOs, existe a indicação de washout de quatorze dias antes da introdução de trazodona (Epocrates). Com agonista $5 \mathrm{HT}_{1}$, há o relato de SS na associação com buspirona (Goldberg e Huk, 1992), triptanos (Epocrates) e tandospirona (Kaneda e Ohmori, 2001). Logo, a combinação de fármacos serotoninérgicos deve ser monitorada. Além do mecanismo farmacodinâmico, com o conhecimento hoje adquirido sobre as ações do metabólito ativo $\mathrm{mCPP}$ e do papel de algumas enzimas do sistema citocromo P450 na metabolização da trazodona, podemos pensar também na possibilidade do envolvimento de interação do tipo farmacocinético. Essa idéia é reforçada pelo achado de elevação de $31 \%$ dos níveis de trazodona com a associação de fluoxetina (Kasper et al., 2005).

Em 2003, Greenblatt et al. pesquisaram em voluntários sadios a associação entre trazodona e ritonavir, droga anti-retroviral, inibidora de CYP 3A4 com conhecido potencial de interação farmacológica. Sua investigação mostrou exacerbação dos efeitos colaterais naqueles que receberam ambos os medicamentos, como aumento de sedação e fadiga associado à elevação da concentração plasmática da trazodona e ao prolongamento de sua meia-vida. Com frequiência, a insônia e a ansiedade estão presentes nos pacientes infectados com o HIV, e a prescrição de trazodona tem sido uma alternativa terapêutica que deve ser monitorada na presença de ritonavir.

A enzima CYP 3A4 está envolvida na etapa de biotransformação da trazodona para mCPP (Weisler et al., 1994). Outras enzimas mostram evidência de algum papel na metabolização da trazodona, como a CYP 2D6 e a CYP 1A2 (Baldessarini, 2006; Kasper et al., 2005). A enzima CYP 2D6 metaboliza o mCPP para p-hidroximCPP, seu principal metabólito (Rotzinger et al., 1999). Segundo Baldessarini (2006), a enzima CYP 2C19 também participa da metabolização do mCPP.

Em um estudo realizado com onze pacientes deprimidos, a trazodona foi prescrita de 1 a 18 semanas, e a tioridazina foi co-administrada por 1 semana; amostras de sangue foram coletadas antes e depois da introdução de tioridazina, e o resultado mostrou clara elevação tanto dos níveis de trazodona quando de seu metabólito ativo, o mCPP (Yasuy et al., 1995).

Devem ser acompanhados com atenção aqueles pacientes que estiverem em uso de trazodona concomitante a fármacos inibidores de CYP 3A4, 2D6 e provavelmente CYP1A2 (Cantarelli e Marcolin, 2003; Cupp e Tracy, 1998).

Casos descritos de forma isolada incluem a possível precipitação de toxicidade pela fenitoína (Dorn, 1986), pela digoxina (Rauch e Jenike, 1984) e desenvolvimento de coma na associação de ginkgo biloba em paciente com diagnóstico de doença de Alzheimer (Galuzzi et al., 2000).

$\mathrm{O}$ imatinibe (usado em pacientes com leucemia mielóide crônica e tumor do estroma gastrointestinal) e a amiodarona podem aumentar os níveis plasmáticos de trazodona, com risco de síncope e hipotensão por metabolismo hepático inibido, enquanto a carbamazepina pode diminuir os níveis de trazodona por indução hepática (Epocrates).

Quadro 2. Enzimas do sistema citocromo P450 envolvidas na metabolização da trazodona e do mCPP

\begin{tabular}{l|c|c}
\hline Indutores* & CYP & \multicolumn{1}{c}{ Inibidores** } \\
\hline $\begin{array}{l}\text { Carbamazepina, efavirenz, fenitoína, } \\
\text { oxcarbazepina, fifampicina, ritonavir }\end{array}$ & $3 A 4$ & $\begin{array}{c}\text { Nefazodona, fluvoxamina, fluoxetina, sertralina, paroxetina, venlafaxina, antifúngicos } \\
\text { azoles, cimetidina, claritromicina, diltiazem, inibidores da protease, eritromicina }\end{array}$ \\
\hline $\begin{array}{l}\text { Cafeína, carbamazepina, lansoprazol, } \\
\text { rifampicina, ritonavir, tabaco }\end{array}$ & 1 A2 & $\begin{array}{r}\text { Paroxetina, fluoxetina, sertralina, fluvoxamina, nefazodona, venlafaxina, } \\
\text { clomipramina, amitriptilina, cimetidina, tioridazina, haloperidol }\end{array}$ \\
\hline $\begin{array}{l}\text { Carbamazepina, fenitoína, } \\
\text { rifampicina, secobarbital }\end{array}$ & 2 Amig & Cetoconazol, cimetidina, cloranfenicol, fluoxetina, efavirenz, paroxetina, ritonavir \\
\hline
\end{tabular}

* Induzem a atividade da enzima, aumentando o metabolismo do substrato.

** Inibem a atividade da enzima, diminuindo o metabolismo do substrato.

Referências: Baldessarini, 2006; Cantarelli e Marcolin, 2003; Cupp e Tracy, 1998; Kalgutkar et al., 2005; Rotzinger et al., 1999; Zalma et al., 2000 


\section{Reações adversas temidas}

\section{Hepatite medicamentosa}

A maioria dos medicamentos pode gerar complicações hepáticas, mas essas reações são pouco frequientes e consideradas idiossincráticas (Lee, 2003). Em geral, acontecem em doses terapêuticas, com um período variável entre 5 e 90 dias do início do tratamento. Ficando clara a relação entre o medicamento e o desenvolvimento do quadro, a interrupção do fármaco é imprescindível para a normalização da função hepática, haja vista a possibilidade de evolução para insuficiência hepática aguda, transplante e/ou óbito.

Vários tipos de alteração hepática podem suceder ao início de uma medicação. Com a trazodona, uma alteração hepatocelular pode comprometer o tratamento, provocada por lesão direta ou ativação enzimática, levando a disfunção celular, de membrana e resposta citotóxica de células T. Outros medicamentos implicados nesse tipo de reação incluem a isoniazida, a lovastatina, a venlafaxina e a nefazodona.

Beck et al. (1993) citam três casos descritos na literatura de dano hepático induzido por trazodona com resolução entre 4 e 24 semanas após a interrupção do tratamento e relatam o desenvolvimento de hepatite crônica ativa em paciente feminina, asiática, com 75 anos, após alguns meses de tratamento com $150 \mathrm{mg} /$ dia de trazodona e confirmado por biópsia hepática. Outro paciente, de 60 anos, com diagnóstico de demência de Pick mostrou alterações de enzimas hepáticas em exames de rotina que foram identificadas após seis meses da introdução de trazodona por quadro de agitação. Os exames foram refeitos 45 dias depois da retirada do fármaco, com normalização das taxas de enzimas hepáticas (Sánchez Ruiz et al., 2004). A causa dessa reação adversa permanece indeterminada, mas a ligação temporal entre o início da medicação e o surgimento do quadro, bem como a melhora com a retirada, mostra uma correlação direta entre causa e efeito. Tais alterações hepáticas podem estar associadas à ativação metabólica da trazodona, similar à que acontece com o acetaminofeno, com formação de metabólitos reativos capazes de causar dano celular (Kalgutkar et al., 2005). Interações farmacológicas que predisponham a isso não foram encontradas na literatura pesquisada.

\section{Alterações de condução cardíaca}

Os baixos efeitos anticolinérgicos da trazodona fizeram desse medicamento uma opção para os ATCs quanto à menor probabilidade de reações adversas cardiovasculares. Apesar disso, encontramos na literatura relatos individuais que vão desde o surgimento de bloqueio cardíaco completo após uma única dose de trazodona em paciente com doença cardiovascular preexistente até a descrição do caso de uma paciente de 29 anos que utilizava fluoxetina ( $40 \mathrm{mg}$ pela manhã), trazodona (50 mg à noite) e diazepam (30 mg), quando necessário, e desenvolveu prolongamento de intervalo QT, mas sem arritmia, após ingestão aguda de $3 \mathrm{~g}$ de trazodona (Levenson, 1999; Rauch e Jenike, 1984; Rausch et al., 1984). Similarmente, foi descrito o desenvolvimento de arritmia ventricular, em doses terapêuticas, tanto em paciente com história de doença cardíaca prévia (Aronson e Hafez, 1986; Janowsky et al., 1983; Vlay e Friedling, 1983) quanto sem (Johnson, 1985). Em pesquisas mais recentes, parece que, assim como outras medicações, a trazodona pode gerar prolongamento de intervalo QT e predispor a arritmias por inibição do fluxo de potássio através dos canais hERG, prejudicando a repolarização celular (Tarantino et al., 2005). O gene mutante hERG codifica o canal $\mathrm{I}_{\mathrm{Kr}}$, corrente delayed rectifier de potássio (ativação rápida), e tem ligação com prolongamento congênito do intervalo QT; geralmente, medicamentos prolongam o intervalo QT por bloquear canais de potássio associados à voltagem e, seletivamente, $\mathrm{I}_{\mathrm{Kr}}$ (Costa $\mathrm{e}$ Gonçalves, 2004).

No ano de 2003, nos Estados Unidos, ocorreram 15,5 milhões de prescrições de trazodona, aproximadamente 11,7 milhões para insônia (Tarantino et al., 2005). Em virtude da sua presença no receituário, devemos ficar atentos para o potencial de interação farmacodinâmica com outras substâncias também relacionadas ao prolongamento de intervalo QT (Prybys, 2004; Rettenbacker et al., 2005; Strain et al., 2004; Straus et al., 2004; Witchel et al., 2003) e que estão listadas no quadro 3.

Quadro 3. Medicações relacionadas ao aumento de intervalo QT

\begin{tabular}{l|l}
\hline Antidepressivos & Quinolonas \\
Amitriptilina & Gatifloxacino \\
Clomipramina & Levofloxacino \\
Desipramina & Moxifloxacino \\
Fluvoxamina & Sparfloxacino \\
Imipramina & Bloqueadores dos canais de cálcio \\
Maprotilina & Diltiazem \\
Nortriptilina & Verapamil \\
Nefazodona & Outros antibióticos \\
Antipsicóticos & Eritromicina \\
Droperidol & Claritromicina \\
Haloperidol & Procinético gastrointestinal \\
Pimozida & Cisaprida \\
Tioridazina & \\
Ziprazidona & \\
Antiarrítmicos & \\
Amiodarona & \\
Procainamida & \\
Quinidina &
\end{tabular}

Referências: Prybys, 2004; Rettenbacker et al., 2005; Strain et al., 2004; Straus et al., 2004; Witchel et al., 2003 


\section{Priapismo}

O priapismo é uma ereção peniana, ou de clitóris, dolorosa e prolongada, podendo durar várias horas (Berk e Acton, 1997; Medina, 2002). Quando ocorre, geralmente é em decorrência de efeito farmacológico, o bloqueio alfa 1 -adrenérgico central é o mecanismo mais comumente implicado nesta reação. Embora incomum, por sua gravidade constitui-se em emergência urológica que deve ser reconhecida, podendo gerar extensa lesão tecidual com risco de impotência permanente. Tem sido descrito com o uso de vários psicofármacos, inclusive a trazodona e o conhecido sildenafil, agente utilizado para disfunção erétil (Correaz-Gomez et al., 2000; Thompson et al., 1990; Wilt e Fink, 2004).

Segundo Thompson et al. (1990), 15\% a 45\% dos casos de priapismo estão relacionados a medicações, e as psicotrópicas são as mais freqüentemente implicadas. Entre os fármacos citados na literatura envolvidos neste processo estão: olanzapina (Bucur e Mahmood, 2004; Childers et al., 2003; Compton et al., 2000; Jagadheesan et $a l ., 2004$; Songer e Barclay, 2001), quetiapina (Bourgeois e Mundh, 2003; Davol e Rukstalis, 2005; Pais e Ayvazian, 2001; Salado et al., 2002), clozapina (Toofanny e Maddens, 2002), clorpromazina (Bucur e Mahmood, 2004; Kilciler et al., 2003; Negin e Murphy, 2005), tioridazina (Bucur e Mahmood, 2004), risperidona (Owley et al.,
2001; Slauson e LoVecchio, 2004), zuclopentixol (Salado et al., 2002) e nefazodona (Toofanny e Maddens, 2002). Também há na literatura priapismo e ereção prolongada na associação entre olanzapina e lítio (Wilt e Fink, 2004), oxcarbazepina, aripiprazol e lítio (Negin e Murphy, 2005), risperidona e lítio (Owley et al., 2001). Logo, a conduta adequada é a identificação de ereções mais prolongadas ou lentificação da detumescência como indicativo de risco para o desenvolvimento de priapismo, tanto para o uso isolado quanto para o associado, e a correta informação ao paciente ou responsável.

\section{Conclusões}

As informações obtidas sobre a trazodona nesta revisão permitem dizer que se trata de um medicamento seguro para o adulto, mostrando-se como opção para o tratamento de quadros associados a sintomas depressivos, ansiosos e com distúrbio do sono. Considerando ainda seu mecanismo de ação e seu padrão de efeitos colaterais e de interação farmacológica, a trazodona representa uma medicação importante no arsenal do tratamento de quadros psiquiátricos no idoso. Dada sua prescrição para várias situações, bem como seu uso freqüente, mesmo que em doses variadas, as pesquisas continuam buscando novas informações.

\section{Referências bibliográficas}

Angelino, A.F.; Treisman, G.J. - Management of psychiatric disorders in patients infected with Human Immunodeficiency Virus. Clin Infec Dis 33: 847-856, 2001.

Aronson, M.D.; Hafez, H. - A case of trazodone-induced ventricular tachycardia. J Clin Psychiatry 47: 388-389, 1986.

BaldesSARINI, R.J. - Drug therapy of depression and anxiety disorders, In: Brunton, L.L.; Lazo, J.S.; Parker, K.L. (eds.) The pharmacological basis of therapeutics - Goodman \& Gilman's. 11.ed. McGrawn Hill, New York, pp. 429-459, 2006.

Beck, P.L.; Bridges, R.J.; Demetrick, D.J.; Kelly, J.K.; Lee, S.S. - Chronic active hepatitis associated with trazodone therapy. Ann Int Med 118: 791-792, 1993.

BeRK, M.; Acton, M. - Citalopram-associated clitoral priapism: a case series. Int Clin Psychopharmacol 12: 121-122, 1997.

Bourgeols, J.A.; MundH, H. - Priapism associated with risperidone: a case report. J Clin Psychiatry 64: 218-219, 2003.

Brogden, R.N.; Heel, R.C.; Speight, T.M.; Avery, G.S. - Trazodone: a review of its pharmacological properties and therapeutic use in depression and ansiety. Drugs 21: 401-429, 1981.

Bucur, М.; Maнm00D, T. - Olanzapine-induced clitoral priapism. J Clin Psychopharmacol 24: 572-573, 2004.

Cantarell, M.G.; Marcolin, M.A. - Interações Medicamentosas, In: Cataldo Neto, A.; Gauer, G.J.C.; Furtado, N.R. (orgs.) Psiquiatria para estudantes de medicina. Edipucrs, Porto Alegre, pp. 757-762, 2003.

Childers, J.B.; Schwartz, A.C.; Compton, M.T. - Olanzapine- associated priapism. Psychosomatics 44: 351-352, 2003.

Compton, M.T.; SAldivia, A.; BerRY, S.A. - Recurrent priapism during treatment with clozapine and olanzapine. Am J Psychiatry 157: 659, 2000.
Correaz-Gomez, M.A.; Portillo Martin, J.A.; Martin Garcia, B.; Hernandez Rodríguez, R.; Gutierrez Banos, J.L.; del Valle Schaan, J.I. et al. - Trazodone-induced priapism. Act Urol Esp 24: 840-842, 2000.

Costa, A.M.N.; GonçaLves, I. - Alterações cardiovasculares induzidas pelo uso de medicações psicotrópicas. Psiq Prática Méd. Disponível em www. aperj.com.br/publicacoes/revista/2004. Acessado em 02.02.2006.

Cupp, M.J.; Tracy, T.S. - Cytochrome P450: new nomenclature and clinical implications. Clin Pharmacology 57: 107-116, 1998.

Davol, P.; RuKSTALIS, D. - Priapism associated with routine use of quetiapine: case report and review of the literature. Urology 66: 880-882, 2005.

Dorn, J.M. - A case of phenytoin toxicity possibly precipitated by trazodone. J Clin Psychiatry 47: 89-90, 1986.

Dugan, D.J. - Antidepressant: using pharmacology to individualize therapy. $J$ Pharm Pract 14: 458-466, 2001.

EPocrates. Disponível em: www.epocrates.com.

Escobar, J.I.; Gomez, J.; Constain, C.; Rey, J.; Santacruz, H. - Controlled clinical trial with trazodone a novel antidepressant. A South American experience. J Clin Pharmacol 124-130, 1980.

Freemantle, N.; Anderson, I.M.; Young, P. - Predictive value pharmacological activity for the relative efficacy of antidepressant drugs. Br J Psychiatry 177: 292-302, 2000.

Galluzzl, S.; Zanetti, 0.; Binettı, G.; Trabucchi, M.; Frisoni, G.B. - Coma in a patient with Alzheimer's disease taking low dose trazodone and ginkgo biloba. J Neurol Neurosurg Psychiatry 68: 679-680, 2000.

George, T.P.; Godleski, L.S. - Possible serotonin syndrome with trazodone addition to fluoxetine. Biol Psychiatry 39: 384-385, 1996.

GoldBERG, R.J.; Huk, M. - Serotonin syndrome from trazodone and buspirone. Psychosomatics 33: 235-236, 1992. 
Golden, R.N.; Dawkins, K.; Nicholas, L. - Trazodone and nefazodone, In: Schatzberg, A.F.; Nemeroff, C.B. (eds.) Textbook of Psychopharmacology. 3.ed. American Psychiatric Publishing, Washington, pp. 315-325, 2004.

Greenblatt, D.J.; von Moltke, L.L.; Harmatz, J.S.; Fogelman, S.M.; Chen, G.; GRAF, J.A. et al. - Short-term exposure to low-dose ritonavir impairs clearance and enhances adverse effects of trazodone. J Clin Pharmacol 43: 414-422, 2003.

HARDY, J.L.; SIRoIS, A. - Reduction of prothrombin and partial thromboplastin times with trazodone. CMAJ 135: 1372, 1986.

Hingtgen, J.N.; Hendrie, H.C.; Aprison, M.H. - Postsynaptic serotonergic blockade following chronic antidepressive treatment with trazodone in an animal model of depression. Pharmacol Biochem Behavior 20: 425-428, 1984.

JagadheESAn, K.; Thakur, A.; AkhtaR, S. - Irreversible priapism during olanzapine and lithium therapy. Aust N Z J Psychiatry 38: 381, 2004

Janowsky, D.; Curtis, G.; Zisook, S.; Kuhn, K.; Resovsky, K.; Le Winter, M. - Ventricular arrhythmias possibly aggravated by trazodone. Am J Psychiatry 140: 796-797, 1983.

Johnson, B.A. - Trazodone toxicity. Br J Hosp Med 33: 298, 1985

KalgutKar, A.S.; Henne, K.R.; Vaz, A.D.; Colın, C.; Sogla, J.R.;Zhao, S.X. etal. - Metabolic activation of the nontricyclic antidepressant trazodone to electrofhilic quinone-imine and epoxide intermediates in human liver microsomes and recombinant P450 3A4. Chem Biol Interact 155: 10-20, 2005

Kaneda, Y.; Ohmori, T. - Possible mild serotonin syndrome related to coprescription of tandospirone and trazodone. Gen Hosp Psychiatry 23: 98-100, 2001.

Kasper, S.; Olivieiri, L.; Di Loreto, G.; Dionisio, P. - A comparative, randomised, doublé-blind study of trazodone prolonged-release and paroxetine in the treatment of patients with major depressive disorder. Curr Med Res Opinion 21: 1139-1146, 2005.

Kilciler, M.; BediR, S.; Sumer, F.; Dayanc, M.; Peker, A.F. - Priapism in a patient receiving long-term chlorpromazine therapy. A case report. Urol Int 71: 127-128, 2003

LACY, T.; MathIS, M. - Dissociative symptoms from combined treatment with sertraline and trazodone. J Neuropsychiatry Clin Neurosci 15 : 241-242, 2003.

LEE, W.M. - Drug-induced hepatotoxicity. N Eng/ J Med 349: 474-485, 2003.

LeVEnson, J.L. - Prolonged OT interval after trazodone overdose. Am J Psychiatry 156: 969-970, 1999.

LieBowitz, N.R.; EL-MaLlaKh, R.S. (letter). - Trazodone for the treatment of anxiety symptoms in substance abusers. $J$ Clin Psychopharmacol 9: 449-451, 1989.

MacHale, S. - Managing depression in physical illness. Adv Psych Treat 8 : 297-306, 2002.

McCuE, R.E.; Joseph, M. - Venlafaxine and trazodone induced serotonin syndrome. Am J Psychiatry 158: 2088-2089, 2001.

Marcol, M.; Maura, G.; Tortarolo, M.; Raiteri, M. - Trazodone is a potent agonist at $5-\mathrm{HT} 2 \mathrm{C}$ receptors mediating inhibition of the $\mathrm{N}$-methyl$\mathrm{D}$-aspartate/nitric oxide-cyclic GMP pathway in rat cerebellum. $J$ Pharmacol Exp Ther 285: 983-986, 1998.

Marek, G.J.; McDougle, C.J.; Price, L.H.; Seiden, L.S. - A comparison of trazodone and fluoxetine: implications for a serotonergic mechanis of antidepressant action. Psychopharmacology 109: 2-11, 1992.

Margolese, H.C.; Chouinard, G. - Serotonin syndrome from addition of lowdose trazodone to nefazodone. Am J Psychiatry 157: 1022, 2000.

Medina, C. - Clitoral priapism: a rare condition presenting as a cause of vulvar pain. Am Coll Obst Gyn 100: 1089-1091, 2002.

NegIN, B.; MuRPhy, T.K. - Priapism associated with oxcarbazepine, aripiprazole and lithium. J Am Acad Child Adolesc Psychiatry 44: 1223-1224, 2005.

NisIJIMA, K.; ShimizU, M.; AвE, T.; IsHiguRo, T. - A case of serotonin syndrome induced by concomitant treatment with low-dose trazodone and amitriptyline and lithium. Int Clin Psychopharmacol 11: 289-290, 1996.

OdAgAKI, Y.; TOYOSHIMA, R.; YAMAUCHI, T. - Trazodone and its active metabolite mchlorophenylpiperazine as partial agonists at $5 \mathrm{HT}$ receptors assessed by [ ${ }^{35}$ S] GTPãS binding. J Psychopharmacol 19: 235-241, 2005.

Owley, T.; LeVenthaL, B.; Cook, E.H. (letter). - Risperidone-induced prolonged erections following the addition of lithium. $J$ Child Adol Psychopharmacol 11: 441-442, 2001.
PaIS, V.M.; AyvazIAn, P.J. - Priapism from quetiapine overdose: first report and proposal of mechanism. Urology 58:462, 2001.

PrYBrs, K.M. - Deadly drug interactions in emergency medicine. Emerg Med Clin N Am 22: 845-863, 2004.

RAUCH, P.K.; JENIKE, M.A. - Digoxin toxicity possibly precipitated by trazodone. Psychosomatics 25: 334-335, 1984.

Rausch, J.L.; Pavlinac, D.M.; Newman, P.E. - Complete heart block following a single dose of trazodone. Am J Psychiatry 141: 1472-1473, 1984.

REEves, R.R.; Bullen, J.A. - Serotonin syndrome produced by paroxetine and low-dose trazodone. Psychosomatics 36: 159-160, 1995.

Rettenbacker, M.A.; Eder-Ischia, U.; Bader, A.; Edlinger, M.; Hofer, A.; Hummer, M. et al. - QTc variability in schizophrenia patients treated with antipsychotics and healthy controls. Journal of Clinical psychopharmacology 25: 206-210, 2005

Rothman, R.B.; Baumann, M.H., Savage, J.E.; Rauser, L.; McBride, A.; Hufeisen, S.J. et al. - Evidence for possible involvement of $5 \mathrm{HT}_{2 \mathrm{~B}}$ receptors in the cardiac valvulopathy associated with fenfluramine and other serotonergic medications. Circulation 102: 2836-2841, 2000.

Rotzinger, S.; Bourin, M.; АкIMoto, Y.; CoutTS, R.T.; BakeR, G.B. - Metabolism of some "second"- and fourth-generation antidepressants: iprindole, viloxazine, bupropion, mianserin, maprotiline, trazodone, nefazodone, and venlafaxina. Cell Mol Neurobiol 19: 427-442, 1999.

RotZIngER, S.; FANG, J.; BAKER, G. - Trazodone is metabolized to m-chlorophenylpiperazine by CYP3A4 from human sources. Drug Metabol Disposition 26: 572-575, 1998

Salado, J.; Blazouez, A.; Diaz-Simon, R.; Lopez-Munoz, F.; Alamo, C.; Rubio, G. - Priapism associated with zuclopenthixol. Ann Pharmacother 36 1016-1018, 2002

Sánchez Ruiz, J.C.; González López, E.; Aparício Tijeras, C.; Ezouerra Gadea, J. - Hepatotoxicidad por trazodona. Aten Primaria 34: 104, 2004.

SLAuSON, S.D.; LoVECCHIO, F. - Risperidone-induced priapism rechallenge. J Emerg Med 27: 89-90, 2004.

Small, N.A.; Giamonna, K.A. - Interaction between trazodone and warfarin. Ann Pharmacother 34: 734-736, 2000.

Songer, D.; BarCLAY, J. - Olanzapina-induced priapism. Am J Psychiatry 158: 2087-2088, 2001.

Stefanini, E.; Fadda, F.; Medda, L.; Gessa, G.L. - Selective inhibition of serotonin uptake by trazodone, a new antidepressant agent. Life Sci 8: 1459-1466, 1976.

SteRnBACH, H. - The serotonin syndrome. Am J Psychiatry 148: 705-713, 1991.

Strain, J.J.; Chiu, N.M.; Sultana, K.; Karim, A.; Caliendo, G.; Mustafá, S. et al. - Psychotropic drug versus psychotropic drug - update. Gen Hosp Psychiatry 26: 87-105, 2004.

Straus, S.M.J.M.; Bleumink, G.S.; Dieleman, J.P.; van Der Lel, J.; Jong, W.; Kingma, H. et al. - Antipsychotics and the risk of sudden cardiac death. Arch Intern Med 164: 1293-1297, 2004

Tarantino, P.; Appleton, N.; LanSDell, K. - Effect of trazodone on hERG channe current and QT-interval. Eur J Pharmacol 510: 75-85, 2005.

Thompson, J.W.; Ware, M.R.; BLASHField, R.K. - Psychotropic medication and priapism: a comprehensive review. J Clin Psychiatry51: 430-433, 1990.

Toofanny, N.; Maddens, M.E. - Reversible Penile priapism associated with nefazodone. J Am Geriatr Soc 50: 1610-1611, 2002.

VLAY, S.C.; Frieduing, S. - Trazodone exacerbation of VT. Am Heart J 106 604, 1983.

Weisler, R.H.; Johnston, J.A.; LineberRY, C.G.; Samara, B.; BranconnieR, R.J.; BıLLow, A.A. - Comparison of bupropion and trazodone for the treatment of Major Depression. J Clin Psychopharmacol 14: 170-179, 1994.

WILT, T.J.; FINK, H.A. - Is antidepressant plus sildenafil a recipe for priapism? Postgrad Med 116: 11-12, 2004

Witchel, H.J.; Hancox, J.C.; Nutr, D.J. - Psychotropic drugs, cardiac arrhythmia, and sudden death. J Clin Psychopharmacol 23: 58-77, 2003

YasuY, N.; Otani, K.; Kaneko, S.; OhKUBo, T.; Osanal, T.; ISHIDA, M. et al. - Inhibition of trazodone metabolism by thioridazine in humans. Ther Drug Monit 17: 333-335, 1995

Zalma, A.; von Moltke, L.L.; Granda, B.W.; Harmatz, J.S.; Shader, R.I.; GreenBLATT, D.J. - In vitro metabolism of trazodone by CYP3A: inhibition by ketoconazole and human immunodeficiency viral protease inhibitors Biol Psychiatry 47: 655-661, 2000. 